\title{
A conceptual model of performance measurement for supply chains
}

\author{
Felix T.S. Chan \\ Department of Industrial and Manufacturing Systems Engineering, \\ The University of Hong Kong, Hong Kong \\ H.J. Qi \\ Department of Industrial and Manufacturing Systems Engineering, \\ The University of Hong Kong, Hong Kong \\ H.K. Chan \\ Department of Industrial and Manufacturing Systems Engineering, \\ The University of Hong Kong, Hong Kong \\ Henry C.W. Lau \\ Department of Industrial and Systems Engineering, \\ The Hong Kong Polytechnic University, Hong Kong \\ Ralph W.L. Ip \\ Department of Manufacturing Engineering and Engineering Management, \\ City University of Hong Kong, Hong Kong
}

\begin{abstract}
Keywords
Supply chain management, Systems analysis, Fuzzy logic, Performance measures

\section{Abstract}

Supply chain management (SCM) has gained a tremendous amount of attention from both industries and researchers since the last decade. Until now, there are numerous papers, articles, and reports that address SCM, but there is still a lack of integration between the existing performance measurement methods and practical requirements for the SCM. An innovative performance measurement method is proposed to provide necessary assistance for performance improvement in SCM. The proposed method will address this purpose in these fou aspects: a simplified supply chain model; tangible and intangible performance measures in multiple dimensions; a cross-organizational performance measurement; and fuzzy set theory and weighted average method.
\end{abstract}

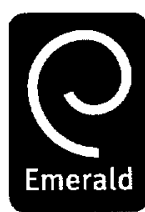

\begin{tabular}{l} 
Management Decision \\
$41 / 7$ [2003] 635-642 \\
\hline MCB UP Limited \\
[ISSN 0025-1747] \\
[DOI $0.1108 / 00251740310495568]$
\end{tabular}

\section{Introduction}

In modern business environments, manufacturers face an increasing pressure of customers' requirements in products customisation, quality improvement, and demand responsiveness. In order to sustain the business under these pressures, more and more enterprises are striving to develop long-term strategic partnerships with a few competent suppliers and collaborate with them in product development, inventory control, and non-core process outsourcing. In addition, the increasing competitive imperatives of cost efficiency and customer responsiveness have pushed firms to pursue strategic alliance with suppliers,

downstream customers, and a host of logistics service providers to exploit their capabilities and create new value to end consumers. This partnership or alliance is known as supply chain and the planning, organizing, and control of the activities in this supply chain is called supply chain management (SCM).

Although SCM has become common practice across all industries, and a steady stream of articles dealing with theories and practices of SCM have been published, the topic of performance measurement of SCM (Beamon, 1999; Gunasekaran et al., 2001). As an indispensable management tool, necessary assistance for performance improvement in pursuit of supply chain excellence. However, many critical drawbacks pervert the existing performance does not receive adequate attention therein performance measurement provides the

measurement systems (PMSs) from making significant contribution to the development and improvement of SCM. With the aim to fill this gap, this paper attempts to propose an innovative performance measurement method for SCM.

Performance measurement is an essential element of effective planning and control, as well as decision making. It can provide necessary feedback information to reveal progress, enhance motivation and communication, and diagnose problems (Waggoner et al., 1999). In SCM context, performance measurement can further facilitate inter-understanding and integration among the supply chain members. The measurement results reveal the effects of strategies and potential opportunities in SCM. There are many publications that have addressed performance measurement in SCM. Beamon (1999) identifies three types of measures: resources, output, and flexibility. Gunasekaran et al. (2001) develop a framework for respectively measuring the performances from strategic, tactical, and operational levels in supply chains; this framework mainly deals with supplier, delivery, customer service, and inventory and logistics costs.

In spite of the importance of performance measurements, there is very little literature available for performance measurement of SCM, especially that deals with system design and measures selection (Beamon, 1999). The contributions of the PMSs in use are discounted by existence of too many flaws in SCM context. Traditional finance-based

The current issue and full text archive of this journal is available at

The Emerald Research Register for this journal is available at http://www.emeraldinsight.com/researchregister http://www.emeraldinsight.com/0025-1747.htm 
Felix T.S. Chan, H.J. Qi, H.K. Chan, Henry C.W. Lau and Ralph W.L. Ip A conceptual model of performance measurement for supply chains

Management Decision

$41 / 7$ [2003] 635-642
PMSs have already received wide criticism on short-term profit orientation, encouraging local optimisation, thus failing to support continuous improvement. Besides, PMSs in SCM context are also accompanied with many problems, as stated below (Gunasekaran et al., 2001):

- being not connected with strategy;

- being lack of balanced approach to integrating financial and non-financial measures;

- being lack of system thinking, in which a supply chain must be viewed as one whole entity, and measured widely across the whole; and

- being loss of supply chain context, and thus encouraging local optimisation.

With these defects aforementioned, an effective performance measurement method has always been under considerable debate, and requires further research exploration.

This paper is organized as follows. This section is the introductory section to SCM and performance measures in SCM. Section 2 is literature review of related area. Section 3 presents the proposed performance measurement method. Section 4 demonstrates the proposed algorithm through a numerical example. Section 5 is conclusion.

\section{Literature review}

Supply chain collaboration benefits in fastening the customer responsiveness, increment of flexibility for changing market conditions, improvement of customer service and satisfaction, and also helps to retain customers. The first initiative of supply chain integration could be dated back to 1992 , when 14 trade association sponsors created a group named "Efficient Consumer Response (ECR) Movement" (Robins, 1994; Barratt and Oliveira, 2001). Then, three years later, five companies: the Benchmarking Partners, Warner-Lambert, Wal-Mart Stores, SAP, and Manaugistics, work on the collaborative planning, forecasting and replenishment (CPFR) project. The goals of the project were to improve the business in the area of forecast accuracy, store in-stock percentage, total inventory (for the retailer and manufacturer combined), and cost (Cooke, 1998). CPFR attempted to bring organizations (retailers and manufacturers) together to make joint plans, including promotion sales, procurement, replenishment, and logistics planning. Blair (1998) stated that CPFR had won the support of organizations in the drug, grocery, general merchandize, and apparel industries.

Researches in SCM are numerous. Barratt and Oliveira (2001) indicated that two significant barriers encountered in implementation of supply chain integration were the lack of visibility of true customer demand, and the collaborative relationships in the area involved joint decision making. New and Ramsay (1997) also stated that there was a danger of unfair distribution of costs and benefits in practice. This might be due to the inequality of power between organizations. Neuman and Samuels (1996) suggested that fairly sharing the gains from the supply chain was critical. Pfohl and Buse (2001) had questioned that what type(s) of organization, in the supply chain, should be the central decision maker to decide part or all of the coordination decisions. Stank et al. (1999) showed that there was a strong support in utilizing automatic inventory replenishment in cross-organizational collaboration.

\subsection{Supply chain performance measures} Anderson et al. (1989) believed that, in measuring logistics performance, a comprehensive strategy of measurement is necessary for the successful planning, realization and control of the different activities, which comprise the business logistics function. Thor (1994) claimed that there should be a family of measures. This is a balanced collection of four to six performance measures, usually including productivity, quality, and customer satisfaction, which, together, furnish an all-inclusive view of results but also provide a diagnostic value. Stainer (1997) believed that a performance measure, or a set of performance measures, is used to determine the efficiency and/or effectiveness of an existing system, or to compare competing alternative systems. Performance measures are also used to design proposed systems, by determining the values of the decision variables that yield the most desirable level(s) of performance. In general, performance measures can be classified as qualitative and quantitative in nature.

\subsubsection{Qualitative performance measures}

Qualitative performance measures are those measures for which there is no direct numerical measurement, although some aspects of them may be quantified. Some examples are as follows:

- Customer satisfaction. The degree to which customers are satisfied with the product and/or service received, and can be applied to internal customers or external customers. Customer satisfaction comprises of three elements; namely, pre-transaction satisfaction, transaction satisfaction, and post-transaction satisfaction.

- Flexibility. The degree to which the supply chain can respond to random fluctuation in the demand pattern. 
Felix T.S. Chan, H.J. Qi, H.K. Chan, Henry C.W. Lau and Ralph W.L. Ip

A conceptual model of

performance measurement for supply chains

Management Decision

$41 / 7$ [2003] 635-642
- Information and material flow integration. The extent to which all functions within the supply chain can pass information and transport materials smoothly.

- Effective risk management. All of the relationships within the supply chain contain inherent risk. Effective risk management describes the degree to which the effect of these risks is minimized.

- Supplier performance. A measurement to describe how good a supplier can deliver raw materials to production facilities on time and in good conditions.

2.1.2 Quantitative performance measures Quantitative performance measures are those measures that may be directly described numerically. Quantitative supply chain performance measures may be categorized by objectives that are based on cost or profit, measures of customer responsiveness, and productivity. Since quantitative measures are something that can be described and handled easy, any qualitative measures should be translated into quantitative measures as many as possible. Some examples of quantitative performance measures are as follows:

1 Measures based on cost:

- Cost minimization. The most widely used objective. Cost is typically minimized for an entire supply chain. One example is to minimize transportation cost.

- Sales maximization. Maximize the amount of sales dollars or units sold.

- Profit maximization. Maximize revenues less costs.

- Inventory investment minimization. Minimize the amount of inventory costs so reduction of the inventory level is required.

- Return on investment maximization. Maximize the ratio of net profit to capital that was employed to produce that profit.

2 Measures based on customer responsiveness:

- Fill rate maximization. Maximize the fraction of customer orders filled on time.

- Product lateness minimization. Minimize the amount of time between the promised product delivery date and the actual product delivery date.

- Customer response time minimization. Minimize the amount of time required from the time an order is placed until the time the order is received by the customer, such as order lead time.

- Lead time minimization. Minimize the time that is required from the time an order has begun its production until the time the order is read for shipment.
- Function duplication minimization. Minimize the number of business functions that are provided by more than one business entity.

3 Measures based on productivity:

- Capacity utilization maximization. Maximize the capacity utilization.

- Resources utilization maximization. Maximize the resources utilization.

\section{An innovative performance measurement method}

In order to solve the mentioned problem, an innovative performance measures evaluation method is introduced in this paper. The proposed method is a mathematical model that employs fuzzy-set theory to measure the integrated performance of complex supply chain. Fuzzy set theory is used to address the real situation in judgment and evaluation processes. It can deal with imprecise information to aid decision making. The procedure of the evaluation is simple: after selection of the appropriate performance measures as mentioned above, data should be collected for analysis. The performance measures are weighted by finding the normalised importance weight through a geometric scale of triangular fuzzy number. Then, fuzzy performance grade is defined to find the fuzzy measurement result and a performance score can be calculated. Finally, the measurement results can be defuzzified to a performance index, which can be used to indicate the performance of the system under evaluation. Details will be presented below.

\subsection{System-thinking perspective and process-based model}

The objective of SCM is to create the most value, not simply for some companies, but for the whole supply chain network, even including the end customers. In this context, successful supply chain measurement systems do more than just focus on partial areas, but rather look across the whole network. This paper suggests that a holistic system-thinking perspective is employed to suit the essence of SCM, in which the supply chain performances should be measured beyond the traditional boundaries of functions and organizations. Thus, a PMS that spans all the key business aspects will facilitate really seamless integration and global optimisation of supply chain performances.

It is much more difficult to manage supply chains than just to define it. Strictly speaking, a supply chain is not a single chain of one-to-one business entities, but rather an inter-related network of multiple members and complex relationships (Lambert et al., 1998). It is common that, in practice, a firm participates in more than 
Felix T.S. Chan, H.J. Qi H.K. Chan, Henry C.W. Lau and Ralph W.L. Ip

A conceptual model of performance measurement for supply chains

Management Decision 41/7 [2003] 635-642 one supply chain, and a firm has not all its functions and divisions involved in one supply chain with the same close integration. This complexity also puzzles performance measurement of supply chains, and therefore necessitates building an effective model to ease the analysis and measurement of SCM.

This paper attempts to build a process based method to simplify the practical supply chains from their essence and commonalities. Generally, a process is a structured set of activities designed to perform specific functions and produce specific outputs. In this simplified model, a process refers to a series of planned activities from original suppliers and manufacturers till retailers add value for the end customers. The core business processes, which are of essential importance to business objectives and strategies, are suggested to identify and confine herein as the framework of this PMS For any supply chain, the general processes and structure can be depicted as shown in Figure 1, in which six core processes are linked. These core processes categorize the typical function areas in supply chains.

The key processes identified can be further decomposed into sub-processes and activities to address their detailed performances. For example, the inbound logistics can be decomposed into such sub-processes as purchasing, transport, supply base management, etc. All of these key processes and sub-processes compose of a hierarchy of supply chain model, which is the framework of the proposed PMS.

\subsection{Appropriate performance measures}

These performances should cover such areas as those:

- of critical concern to supply chain common goals and strategies;

- of inter-influence and of common concern among the supply chain partners; and

- concerned by both internal partners and external customers.
This performance measurement method is based on the process model of the supply chain, so the measures can be derived from process performance.

Any process consumes particular enterprise resources, performs the planned missions and functions, and then adds value to products that are delivered to end customers. The consumed resources, and planned functional operations or expected outcomes are the essential performance of processes. Time, labour, capital, power, facilities, and information are typically the resources that processes consume.

Traditionally, they can be measured in terms of their amount per output unit. The performance on functional operations and expected outcomes can be measured according to their planned functional operations. For example, purchasing process is mainly responsible for material replenishment, supply base management, etc. Thus it can be measured from such performance as material replenishment reliability and quality, and supplier-buyer relationship. Reliability in delivery and transportation, and flexibility in material supply, production, and order delivery have received more and more attention in performance measurement of supply chains.

For each process and its sub-processes that need to be measured, the corresponding measures are identified and grouped into the processes and measures hierarchy (PMH) as shown in Figure 2. This forms the framework of the PMS.

\subsection{Teamwork of performance measurement}

In this research, a performance measurement team (PMT) is suggested. This PMT is composed of the representatives from various areas of the supply chain, which can be shopfloor operators, process supervisors, department directors, and plant managers. The members of PMT serve mainly as the evaluators, and provide wide opinions for each performance. They come from various

\section{$\overline{\text { Figure } 1}$}

General flow chart of supply chain

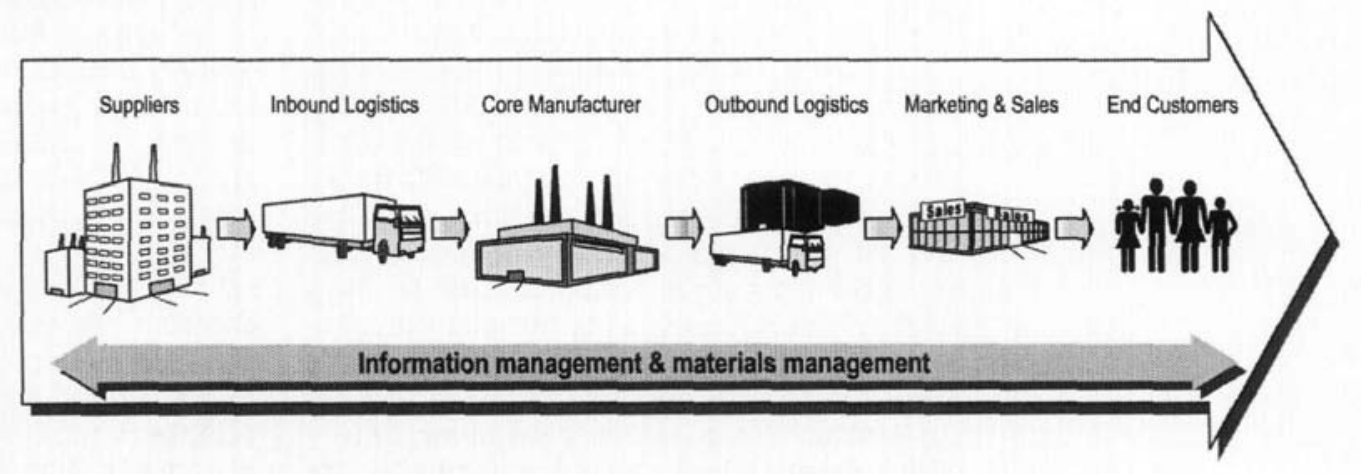


Felix T.S. Chan, H.J. Qi, H.K. Chan, Henry C.W. Lau and Ralph W.L. Ip A conceptual model of performance measurement for supply chains

Management Decision $41 / 7$ [2003] 635-642

\section{$\overline{\text { Figure } 2}$}

General structure of the $\mathrm{PMH}$

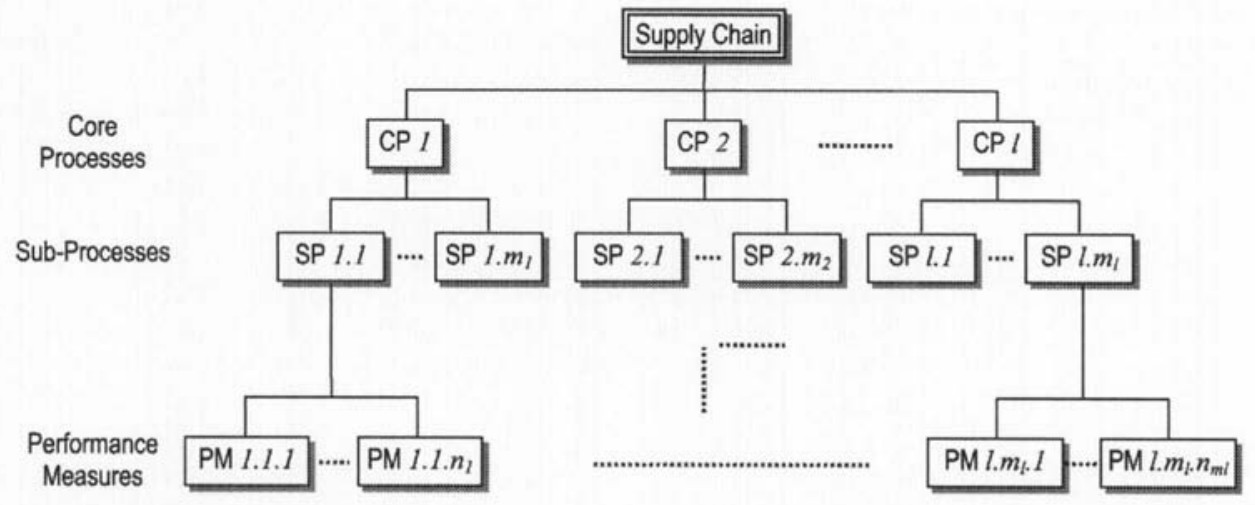

management areas and have a wide background and experiences, and thus can cover a wide range of views. At the same time, the relative weights of their opinions are not necessarily equal to each other. When incorporating their opinions, the weights will be assigned. There are a variety of ways to derive the weights; for example, by top officials of the supply chain, or averaging the viewpoints of PMT members.

\subsection{The new measurement algorithm}

This section is dedicated to outline the measurement algorithm and application of measurement results. The record data that indicate various performances are compared against the measurement scales that are set by the PMT through assessing the corresponding performance goals and histories, as well as the operation environments. A performance grade in forms of fuzzy set is obtained to denote the result of each performance measure. All the measurement results of various measures by each PMT member are incorporated with the weighted averaging aggregation method. The aggregated results in forms of fuzzy sets are defuzzified and used to assess the supply chain performance innovatively and objectively.

\subsubsection{Relative weights of processes and measures}

Due to the changing objectives and strategies of supply chains, the priorities of individual processes and their various dimensions of performance should differ from each other in performance measurement. Accordingly, it is necessary to set relative weights for them to aggregate the measurement results. There naturally contains essential fuzziness and ambiguity in human judgments. Traditional comparison ratio scale with crisp numbers fails to address the fuzziness. Till now, a multitude of literatures have proposed new applications by using fuzzy set theory, in which fuzzy ratios are used instead of crisp ratios. This paper suggests a geometric scale of triangular fuzzy numbers (Boender et al., 1989).

\subsubsection{Measurement scale and fuzzy} performance grade

After getting the relative importance weights of each performance measures, it is still meaningless to judge and assess any performance without its associated context about objectives and histories. Moreover, the existing measurement methods, in which the current performance are measured through simply being divided by the expectations, have such flaws as ignoring operation context, and losing the important information arising from the uncertainty of human judgment. This paper designs a measurement scale and fuzzy performance grade to address these problems.

When measuring one particular performance, the evaluators of the PMT consider the planned goal and history, as well as the associated operation environments, and then set the measurement scale in forms of the interval, ranging from the just acceptable bottom of performance to the totally satisfactory performance. The influence of the associated operation environment and supply chain context being assessed and taken into consideration, the acceptable bottom and totally satisfactory performance are not necessarily same to performance history and goal, respectively.

The judgment process of measurement scales contains fuzziness as well. Therefore, the measurement results are obtained and denoted by fuzzy numbers through these steps: first, comparing current performance against their measurement scales, and then denoting with the crisp numbers ranging from zero to ten as script marking method does; second, mapping the obtained crisp number into fuzzy performance grade set. The performance set in the form of a fuzzy vector $G=\{A, B, C, D, E, F\}$. These six grades $A, B, C, D, E, F$ denote the gradational 
Felix T.S. Chan, H.J. Qi, H.K. Chan, Henry C.W. Lau and Ralph W.L. Ip

A conceptual model of performance measurement for supply chains

Management Decision

$41 / 7$ [2003] 635-642 measurement results ranging from the perfect to the worst. All these grades are defined by the triangular fuzzy numbers as follows (Figure 3):

$$
\begin{array}{cc}
\tilde{A}=T(8,10,10), & \tilde{B}=T(6,8,10), \\
\tilde{C}=T(4,6,8), & \tilde{D}=T(2,4,6), \\
\tilde{E}=T(0,2,4), & \tilde{F}=T(0,0,2) .
\end{array}
$$

This performance grade set $P_{G}(\mu)$ in the finite universe of discourse $G=\{A, B, C, D, E, F\}$ is defined by a set of ordered pairs as follows (Zadeh, 1965):

$$
P_{G}(\mu)=\left\{\left(p_{X}(\mu), \mu\right), X=A, B, \ldots, F\right\},
$$

where $p_{X}(\mu): G \rightarrow[0,1]$, is a mapping called the membership function of the fuzzy set $G$, and $p_{X}(\mu)$ indicates the degree of belongingness or membership value of $\mu$ in $G$. Herein, $\mu$ is the crisp number in comparison of current performance against measurement scales.

Physically, the gradational grades represent the quantification of the degree to which a particular performance satisfies the performance criteria by the PMT. Each evaluator only needs to assess the performance goals and histories as well as considering the influence of the associated operation environments, and then independently figures out its measurement scale.

\subsubsection{Aggregating and defuzzifying the measurement results}

In measurement activity, the opinions of all the evaluators in the PMT are required to incorporate; in order to get the overall picture of one process the measurement results of individual measures and its sub-processes are required to incorporate as well. As mentioned above, due to the different background and familiarity in various areas, the opinions of the evaluators from differing areas should be given different weights. The relative weights of various performance measures and sub-processes can be derived from the changing supply chain objectives and strategies. Both these two aggregation processes can adopt weighted averaging method, i.e. multiplying measurement result matrix with weight

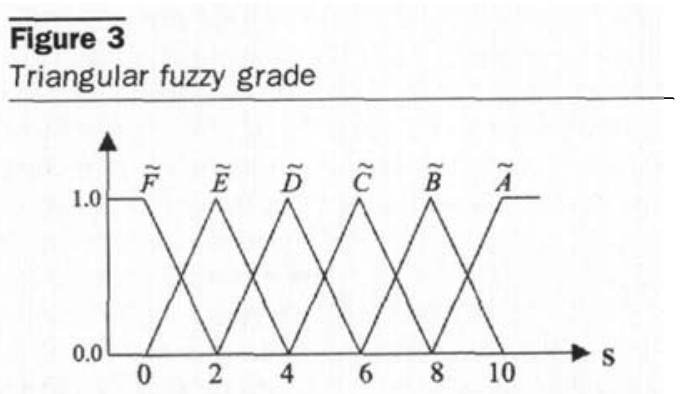

vector. Finally, these results are performance grades in forms of fuzzy sets and cannot be concluded unless being defuzzified into crisp value.

According to their definitions, these gradational performance grades that denote the measurement results represent the meanings of approximation "about". That is, these six grades denote "about ten", "about eight", ... , and "about zero". In this connection, these performance grades can be defuzzified into the crisp numbers from ten to zero through weighted averaging the grade set $P_{G}(\mu)$. The defuzzified crisp number, called performance index, indicates the synthetic assessment of the holistic performance of various areas of supply chains by the evaluator team.

\subsection{The applications of the performance index}

The performance index is a single, global, integrated process performance score.

According to the design of this performance measurement method, this integrated result is the weighted integrative assessment of the whole picture of the supply chain process performance with the multi-dimensions. This simple number provides a concise means to analyse and benchmark the performances in the supply chain systems for their managers. Put in the full range $[0,10]$, this result can be benchmarked. Because the measurement result of each process on the higher layer is aggregated from the measurement results of sub-processes, the worst results can be tracked layer by layer in the PMH. Thus the strengths and weaknesses of supply chain processes are identified and located.

Moreover, the parallel processes can be compared and the problematic nodes can be discovered. Second, from a dynamic sense, the performances of all the key business processes of the supply chain system can be recorded and benchmarked on the base of a monthly or quarterly period. With a series of measurement results at regular intervals, the trend of performance of each business process can be analysed and irregular performance or unsatisfactory progress can be revealed clearly. This information can help managers find and diagnose the problems in the management of supply chain processes.

\section{Numerical example}

For the sake of better illustration of the measurement method proposed above, a simple example is given in this section. Assume cost is one of the measures identified in a supply chain network to indicate its performances and there are four evaluators in the PMT with the relative weights 
Felix T.S. Chan, H.J. Qi H.K. Chan, Henry C.W. Lau and Ralph W.L. Ip A conceptual model of performance measurement for supply chains

Management Decision $41 / 7$ [2003] 635-642
$W^{T}=(0.45,0.25,0.20,0.10)$ of their respective opinions. Please note the sum of the weights should be equal to one, as they should be normalized before putting into this equation.

First, one evaluator makes his judgment for the measure. Assume that the performance history of this cost is $\$ 21$ per unit, and the performance goal requires this cost to be reduced to $\$ 18$ per unit. The evaluator first determines the measurement scale of cost, to assume which is the interval as $[20,18]$ (for this performance, the smaller the better). Suppose the current performance on production cost is average $\$ 19.44$ per unit according to the daily operation records (refer to Figure 3), the performance score and performance grades are calculated as follows

Performance score:

$$
\frac{20-19.44}{20-18} \times(10-0)=2.8 .
$$

Performance grades:

$$
\begin{gathered}
P_{A}(2.8)=0, \quad P_{B}(2.8)=0, \quad P_{C}(2.8)=0 \\
P_{D}(2.8)=\frac{2.8-2}{4-2} 0.4, \quad P_{E}(2.8)=\frac{4-2.8}{4-2}=0.6 \\
P_{F}(2.8)=0 .
\end{gathered}
$$

Thus, the performance grade set can be written as follows:

$$
P_{G}(2.8)=\frac{0}{A}+\frac{0}{B}+\frac{0}{C}+\frac{0.4}{D}+\frac{0.6}{E}+\frac{0}{F},
$$

or

$$
P_{1}^{T}\left(\mu_{1}\right)=(0,0,0,0.4,0.6,0) .
$$

This is the measurement result of cost judged by the first evaluator. For simplicity, assume the performance grade sets by the other three evaluators, in forms of row vector, to be:

$$
\begin{aligned}
& P_{2}^{T}\left(\mu_{1}\right)=(0,0.27,0.73,0,0,0), \\
& P_{3}^{T}\left(\mu_{1}\right)=(0,0,0.31,0.69,0,0), \\
& P_{4}^{T}\left(\mu_{1}\right)=(0,0,0.48,0.52,0,0) .
\end{aligned}
$$

These four vectors compose the fuzzy performance grade matrix as follow: $P\left(\mu_{1}\right)=\left[\begin{array}{llll}P_{1}\left(\mu_{1}\right), & P_{2}\left(\mu_{1}\right), & P_{3}\left(\mu_{1}\right), & P_{4}\left(\mu_{1}\right)\end{array}\right]$

$$
=\left[\begin{array}{cccc}
0 & 0 & 0 & 0 \\
0 & 0.27 & 0 & 0 \\
0 & 0.73 & 0.31 & 0.48 \\
0.4 & 0 & 0.69 & 0.52 \\
0.6 & 0 & 0 & 0 \\
0 & 0 & 0 & 0
\end{array}\right] .
$$

Then the measurement results of these four evaluators, with their relative weights, are aggregated as follows:

$$
P_{1}=P\left(\mu_{1}\right) \cdot W
$$

$$
=\left[\begin{array}{cccc}
0 & 0 & 0 & 0 \\
0 & 0.27 & 0 & 0 \\
0 & 0.73 & 0.31 & 0.48 \\
0.4 & 0 & 0.69 & 0.52 \\
0.6 & 0 & 0 & 0 \\
0 & 0 & 0 & 0
\end{array}\right] \cdot\left[\begin{array}{c}
0.45 \\
0.25 \\
0.20 \\
0.10
\end{array}\right]
$$

$$
=\left[\begin{array}{llllll}
0, & 0.0675, & 0.2925, & 0.3700, & 0.2700, & 0
\end{array}\right]^{T} .
$$

From the mathematical sense, this vector denotes the aggregated opinion of the measurement of cost performance by the four evaluators. It takes the form of fuzzy performance grade set. The performance index is:

$$
\begin{gathered}
P I=\frac{10 \times 0+8 \times 0.0675+6 \times 0.02925}{+4 \times 0.37+2 \times 0.27+0 \times 0} \\
0.0 .675+0.2925+0.3700+0.2700+0 \\
=4.315 .
\end{gathered}
$$

Obviously, 4.315 is not a satisfactory number with respect to the ten-point scale. The supply chain network should be refined in order to improve this performance.

Similarly, if there are other performance measures defined by the evaluators, the individual performance index can be calculated through the same procedures.

\section{Conclusions}

This paper reveals the key issues in the existing performance measurement method especially in SCM context. In order to support performance improvement in SCM, this paper proposes a cross-organizational performance measurement method from a system perspective.

Process-based model, appropriate performance measures, teamwork evaluation, and fuzzy measurement algorithm are outlined, and some suggestions are given. All these designs support comprehensive measurement of the holistic performances of supply chains. In particular, the introduction of fuzzy set theory in setting weights and measuring performances is advantageous, because this fuzzy method addresses the real situation of human judgment with fuzziness in measurement activity without losing the important information as crisp method does. The concise defuzzified results provide easy access to benchmark the performances and avoid excessive proliferation of data.

The major contribution of the proposed methodology is to provide a simple, and robust mathematical model to calculate a performance index of a performance measure in a supply chain network to deal with both tangible and intangible performance measures. The model incorporates fuzzy set 
Felix T.S. Chan, H.J. Qi H.K. Chan, Henry C.W. Lau and Ralph W.L. Ip

$A$ conceptual model of performance measurement for supply chains

Management Decision 41/7 [2003] 635-642 theory, which can represent uncertainties in real-life applications. This method can be employed to replace a traditional pairwise comparison method such as Analytic Hierarchy Process (AHP) (Saaty, 1980) to derive relative weights among the hierarchy. In fact, Saaty's pairwise comparison creates the very unbalanced scale of weight. Though the discrete ratio of (1/9):9 takes the advantage of simplicity, it denies the uncertainties associated with the mapping of human perception and judgment to a crisp number. With the proposed performance measurement method, supply chain managers can easily benchmark the performances of the whole system, and then analyse the effectiveness of their strategies, and identify the potential opportunities. All this feedback information facilitates more objective decision making and performance improvement in SCM.

To optimise the supply chain model, more than one performance measure should be considered. However, a question should be answered first: how to consolidate all qualitative and quantitative performance measures to form a meaningful figure to aid decision making? This is a multi-criteria decision-making problem. Generally, two techniques can be employed for the evaluation; namely, mathematical optimisation and simulation. Mathematical optimisation techniques include exact algorithms that are guaranteed to find an optimal solution. Also, heuristic algorithms can be used to find good solutions, but not necessarily the optimal solutions. This leads to the future research direction of the current study by consolidating all performance measures through similar algorithms so that a single performance index can be found to represent the whole network, regardless of individual performance index.

\section{References}

Anderson, P., Aronson, H. and Storhagen, N.G (1989), "Measuring logistics performance", Engineering Costs and Production Economics, Vol. 17 , pp. 253-62.

Barratt, M. and Oliveria, A. (2001), "Exploring the experiences of collaborative planning initiatives", International Journal of Physical Distribution \& Logistics Management, Vol. 31 No. 4, pp. 266-89.

Blair, N. (1998), "Minding the store: with inventory reduction measures under way in the warehouse, executives are eyeing similar strategies based on store-level data", Supermarket News, Vol. 48 No. 3, pp. 81-2.
Beamon, M.B. (1999), "Measuring supply chain performance", International Journal of Operations \& Production Management, Vol. 19 No. 3, pp. 275-92.

Boender, C.G.E., de Graan, J.G. and Lootsma, F.A. (1989), "Multi-criteria decision analysis with fuzzy pairwise comparisons", Fuzzy Sets and Systems, Vol. 29, pp. 133-43.

Cooke, J.A. (1998), "Into the great wide open", Logistics Management and Distribution Report, Vol. 37 No. 10, pp. 84-7.

Gunasekaran, A., Patel, C. and Tirtiroglu, E. (2001), "Performance measurement and metrics in a supply chain environment", International Journal of Operations \& Production Management, Vol. 21 No. 1/2, pp. 71-87.

Lambert, D.M., Cooper, M.C. and Pagh, J.D. (1998), "Supply chain management: implementation issues and research opportunities", The International Journal of Logistics Management, Vol. 9 No. 2, pp. 1-19.

Neuman, J. and Samuels, C. (1996), "Insights from industry supply chain integration: vision or reality", Supply Chain Management: An International Journal, Vol. 1 No. 2, pp. 7-10.

New, S. and Ramsay, J. (1997), "A critical appraisal of aspects of the lean chain approach", European Journal of Purchasing \& Supply Management, Vol. 3 No. 2, pp. 93-102.

Pfohl, H.C. and Buse, H.P. (2001),

"Inter-organizational logistics systems in flexible production networks, an organizational capabilities perspective", International Journal of Physical Distribution $\&$ Logistics Management, Vol. 30 No. 5, pp. 388408.

Robins, G. (1994), “Sailing into ECR's uncharted waters", Stores, Vol. 76 No. 10, pp. 43-4.

Saaty, T.L. (1980), The Analytical Hierarchy Process, McGraw-Hill, New York, NY.

Stainer, A. (1997), "Logistics - a productivity and performance perspective", Supply Chain Management, Vol. 2 No. 2, pp. 53-62.

Stank, T.P., Daugherty, P.J. and Autry, C.W. (1999), "Collaborative planning: supporting automatic replenishment programs", Supply Chain Management: An International Journal, Vol. 4 No. 2, pp. 75-85.

Thor, C.G. (1994), Measures of Success - Creating a High Performing Organization, Oliver Wight, Essex Junction, VT.

Waggoner, D.B., Neely, A.D. and Kennerley, M.P. (1999), "The forces that shape organizational performance measurement systems: an interdisciplinary review", International Journal of Production Economics, Vol. 60, pp. 53-60.

Zadeh, L.A. (1965), "Fuzzy sets", Information and Control, Vol. 8, pp. 338-56. 